\title{
Neural and cognitive modeling with networks of leaky integrator units
}

\author{
Peter beim Graben ${ }^{1,2}$, Thomas Liebscher ${ }^{3}$, and Jürgen Kurths ${ }^{1}$ \\ 1 Institut of Physics, Nonlinear Dynamics Group, Universität Potsdam, Germany \\ peter@ling. uni-potsdam.de \\ 2 Bundeskriminalamt, Wiesbaden, Germany \\ 3 Institute of Linguistics, Universität Potsdam, Germany
}

Summary. After reviewing several physiological findings on oscillations in the electroencephalogram (EEG) and their possible explanations by dynamical modeling, we present neural networks consisting of leaky integrator units as an universal paradigm for neural and cognitive modeling. In contrast to standard recurrent neural networks, leaky integrator units are described by ordinary differential equations living in continuous time. We present an algorithm to train the temporal behavior of leaky integrator networks by generalized backpropagation and discuss their physiological relevance. Eventually, we show how leaky integrator units can be used to build oscillators which may serve as models of brain oscillations and cognitive processing.

\section{Introduction}

The electroencephalogram (EEG) measures the electric potential of the brain that is generated by large formations of certain neurons, the pyramidal cells. These nerve cells roughly possess an axial symmetry and they are aligned in parallel perpendicular to the surface of the cortex $[14,55,70]$. They receive excitatory input at the superficial apical dendrites from thalamic relay neurons and inhibitory input at the basal dendrites and at their somata from local interneurons $[14,55,21,55,70]$. Excitatory and inhibitory synapses cause different ion currents through the cell membranes thus leading to either depolarization or hyperpolarization, respectively. When both types of synapses are simultaneously active, a single pyramidal cell behaves as a microscopic electric dipole surrounded by its characteristic electrical field $[14,46]$.

According to the inhomogeneity of the cortical gray matter a mass of approximately 10,000 synchronized pyramidal cells form a dipole layer whose fields sum up to the so-called local field potentials that polarize the outer tissues of the scalp thereby acting as a low pass filter [14, 21, 46, 55]. These filtered sum potentials are macroscopically measurable as the EEG at the surface of a subject's head. 
One of the most obvious features of the EEG are oscillations in certain frequency bands. The alpha waves are sinusoidal-like oscillations between 8 $13 \mathrm{~Hz}$, strongly pronounced over parietal and occipital recording sites which reflect a state of relaxation during wakefulness, with no or only low visual attention. Figure 1 shows a characteristic power spectrum for the alpha rhythm: There is one distinguished peak superimposed to a $1 / f$ background EEG. When a subject starts paying attention, the powerful slow alpha waves disappear while smaller oscillations with higher frequencies around $14-30 \mathrm{~Hz}$ (the beta waves) arise [5, 17, 59]. We will refer to this finding, sometimes called desynchronization of the EEG, as the alpha blocking [5]. ${ }^{4}$

Alpha waves are assumed to be related to awareness and cognitive processes $[48,6,39,57]$. Experimental findings suggest that thalamocortical feedback loops are involved in the origin of the alpha EEG [10, 14, 17, 58, 59, 70].

Modeling brain rhythms has a long tradition. Wilson and Cowan [65] were the first who used populations of excitatory and inhibitory neurons innervating each other (see Sect. 3.2). They introduced a two-dimensional state vector whose components describe the proportion of firing McCulloch-Pitts neurons [45] within a unit volume of neural tissue at an instance of time. This kind of ensemble statistics leads to the well-known sigmoidal activation functions for neural networks [30] through the probability distributions of either synapses or activation thresholds (see also [21]). The model further includes the refractory time in which a neuron that has been activated just before cannot be activated again, and the time coarse of postsynaptic potentials as pulse response functions. This model has been strongly simplified by Wilson [64] leading to a network of only two recurrently coupled leaky integrator units (see Sec. 2). Wilson reported limit cycle dynamics of this system for a certain range of the excitatory input playing the role of a control parameter. However, this network does not exhibit an equivalent of the alpha blocking, because the frequency of the oscillations becomes slower for increasing input.

Da Silva et al. [15] pursued two different approaches: a distributed model of the thalamus where relay cells and interneurons are considered individually and a "lumped" model analogous to the one of Wilson and Cowan [65] but without refractory time and even more complicated postsynaptic potentials. In order to determine the sum membrane potential of each population as a model EEG one has to compute the convolution integral of the postsynaptic potential pulse response functions with the spike rate per unit of volume. Linearizing the activation functions allows a system theoretic treatment of the model by means of the Laplace transform and thus computing the power spectrum analytically (see Sec. 4). Da Silva et al. $[15,16]$ have shown that

\footnotetext{
${ }^{4}$ The term "desynchronization" is misleading since it has no direct relation to synchronziation in the sense of e.g. [3, 2]. From the viewpoint of data analysis it simply means: decreasing power in the alpha band of the spectrum. However, the biophysical theories of the EEG explain the loss of spectral power by a loss of coherence of neuron activity, i.e. less synchronization $[5,59,57]$.
} 
their model of thalamical or cortical feedback loops actually exhibits a peak around $10 \mathrm{~Hz}$, i.e. alpha waves, in the spectrum. Though, they were also not able to demonstrate the alpha blocking.

Later, Rotterdam et al. [60] generalized that model to spatio-temporal dynamics by considering a chain of coupled cortical oscillators. A similar approach has been pursued by Wright and Liley $[67,66]$ who discussed a spatial lattice of coupled unit volumes of excitatory and inhibitory elements obeying cortical connectivity statistics. The convolution integrals of the postsynaptic potentials with the spike rates were substituted by convolution sums over discrete time. The most important result which we shall appreciate here, is that the power spectrum shows the alpha peak, and, that there is a "shift to the right" (towards the beta band) of this peak with increasing input describing arousal, i.e. the alpha blocking.

Additionally, Liley et al. [43] suggested also a distributed model of cortical alpha activity using a compartmental description of membrane potentials [40]. In such approach, nerve cells are thought to be built up by cylindrical compartments that are governed by generalized Hodgkin-Huxley equations [31]. Liley et al. [43] reported two oscillatory regimes of this dynamics: one having a broad-band spectrum with a peak in the beta range and the other narrowly banded with a peak around the alpha frequency.

We shall also appreciate the field theoretic models of neural activity $[35,34,68,69,51]$. In these theories the unit volumes of cortical tissue are considered being infinitesimally small. Therefore the systems of coupled ordinary differential equations are substituted by nonlinear partial differential equations. Robinson et al. [51] have proposed such a theory in order to describe thalamocortical interactions and hence the alpha EEG.

Another approach that could lead to the explanation of the EEG is Hebb's concept of a cell assembly [28] where reverberatory circles form neural oscillators. We shall see in Sect. 4.3 how such cycles may emerge in an evolving network.

On the other hand, Kaplan et al. [37] and Smolensky and Legendre [54] argued how neural networks could bridge the gap between the sub-symbolic representation of single neurons and "a symbol-like unit of thought" in models of cognitive processes. Kaplan et al. proposed the cell assembly as an assembly of neural units that are recurrently connected to exhibit reverberatory circles in which information needs to cycle around until the symbolic meaning is fully established. They presented a series of experiments in which they made use of physiological principles that should be present in the functioning of cell assemblies: temporally structured input, the dependency on prior experience, competition between assemblies and control of its activation. A main result is that after a cell assembly is provided with input, its activation gradually increases until an asymptotic activation is reached or the input is distracted. After distraction of the input the activation gradually decreases until it comes back to its resting level. 


\section{Leaky Integrator Networks}

\subsection{Description of leaky integrator units}

When the neural signals are exchanged between different cell assemblies that are typically involved in brain functions, oscillations due to the recurrent connections between the neurons should become visible. A possible way to model this behavior is by describing each cell assembly by a leaky integrator unit, that integrates input over time while the internal activation is continuously decreased by a dampening leakage term. We shall present the relationship between cell assemblies and leaky integrator units in Sec. 3.2. However, also single neurons can be described by a leaky integrator unit, though with quite different leakage constants, as we shall see in Sec. 3.1. In terms of standard units (as e. g. used by Rumelhard et al. [53] a leaky integrator unit looks like the one depicted in Fig. 2.

The activation of this leaky integrator unit is described by

$$
\begin{aligned}
\frac{\mathrm{d} x_{i}(t)}{\mathrm{d} t} & =-x_{i}(t)+\left(1-\alpha_{i}\right) x_{i}(t)+\alpha_{i} f\left(y_{i}(t)\right) \\
& =-\alpha_{i} x_{i}(t)+\alpha_{i} f\left(y_{i}(t)\right) \\
\tau_{i} \frac{\mathrm{d} x_{i}(t)}{\mathrm{d} t}+x_{i}(t) & =f\left(y_{i}(t)\right) .
\end{aligned}
$$

The symbols have the following meanings:

$\frac{\mathrm{d} x_{i}(t)}{\mathrm{d} t}$
$x_{i}(t)$
$y_{i}(t)$
$\alpha_{i}$
$\tau_{i}=\alpha_{i}^{-1}$
$f$

Change of activation of unit $i$ at time $t$ Activation of unit $i$ at time $t$ Netinput of unit $i$ at time $t$ Leakage rate of unit $i$ Time constant of unit $i$ Activation function of each unit; usually sigmoidal-shaped (e.g., logistic as in Eq. (5)), or linear.

The leakage rate $\alpha_{i}$ tells how much a unit depends on the actual netinput. Its value is between 0 and 1 . The higher the value of $\alpha$, the stronger the influence of the previous level of activation and the less the influence of the actual netinput. $\alpha=1$ means that the previous activation doesn't have any influence and the new activation is only determined by the netinput (this is the case e. g. for the standard units used by the PDP group [53]). $\alpha=0$ means that the actual netinput does not have any influence and the activation remains constant. ( $1-\alpha$ could also be regarded as the strength of its memory with respect to earlier activations.)

The netinput of unit $i$ is given as the sum of all incoming signals:

$$
y_{i}(t)=\sum_{j} w_{i j} x_{j}(t)+b_{i}+I_{i}^{e x t}(t) .
$$

With 
$y_{i}(t) \quad$ Netinput of unit $i$ at time $t$

$w_{i j} \quad$ Weight of connection from unit $j$ to unit $i$

$b_{i} \quad$ Bias of unit $i$

$I_{i}^{e x t} \quad$ external Input to unit $i$

Equation (1) is very similar to the general form of neural networks equations for continuous-valued units (described e. g. in [30]). The difference lies in the presence of the leakage term $\alpha$ that makes the current activation dependent from its previous activation. We motivate Eq. (1) by the equivalent recurrent network of Fig. 2 and we shall use it in Sec. 2.2 subsequently to derive a generalized backpropagation algorithm as a learning rule for temporal patterns. On the other hand, Eq. (2) is well-known from the theory of ordinary differential equations. Its associated homogeneous form

$$
\tau_{i} \frac{\mathrm{d} x_{i}}{\mathrm{~d} t}+x_{i}=0
$$

describes simply an exponential decay process. Therefore, the inhomogeneous Eq. (2) can be seen as a forced decay process integrating its input at the right hand side.

Hertz et al. [30, p. 54] discuss a Hopfield network of leaky integrator units which is characterized by Eq. (2) with symmetric synaptic weights $w_{i j}$. Such network is a dynamical system whose attractors are the patterns which are to be learned. Moreover, Hertz et al. [30, p. 55] consider another dynamical system

$$
\tau_{i} \frac{\mathrm{d} x_{i}(t)}{\mathrm{d} t}+x_{i}(t)=\sum_{j} w_{i j} f\left(x_{j}(t)\right)+b_{i}+I_{i}^{e x t}(t)
$$

having the same equilibrium solutions as Eq. (2). As we shall see in Sec. 3.1, Eq. (4) appropriately models small networks of single neurons. However, given a proper activation function $f_{a c t}$, the range of possible activations is resticted to a bounded interval (e. g. ]0,1[ for a logistic function). This leads to a bounded phase space and accordingly is easier to use for proofs [64] or numerical simulations. The time course of activation for a leaky integrator unit using a logistic activation function

$$
f(x)=\frac{1}{1+e^{-\beta x}}
$$

with respect to input and leakage rate is shown in Figs. 3 (a) and (b).

\subsection{Training leaky integrator networks}

In order to use leaky integrator units to create network models for simulation experiments, a learning rule that works in continuous time is needed. The following formulation is motivated by [47] and describes how a backpropagation algorithm for leaky integrator units can be derived. 
In a first step, Euler's method is used to change from differential equations to difference equations:

$$
\begin{aligned}
x_{i}(t+\Delta t) & \approx x_{i}(t)+\frac{\mathrm{d} x_{i}(t)}{\mathrm{d} t} \Delta t \\
\Rightarrow \quad \frac{\mathrm{d} x_{i}(t)}{\mathrm{d} t} & \approx \frac{x_{i}(t+\Delta t)-x_{i}(t)}{\Delta t} .
\end{aligned}
$$

Combining Eq. (1) and Eq. (6) yields

$$
\begin{aligned}
\widetilde{x}_{i}(t+\Delta t) & =(1-\Delta t) \widetilde{x}_{i}(t)+\Delta t\left\{\left(1-\alpha_{i}\right) \widetilde{x}_{i}(t)+\alpha_{i} f\left(\widetilde{y}_{i}(t)\right)\right\} \\
& =\left(1-\Delta t \alpha_{i}\right) \widetilde{x}_{i}(t)+\Delta t \alpha_{i} f\left(\widetilde{y}_{i}(t)\right)
\end{aligned}
$$

where tildes above variables $(\widetilde{x})$ denote continuous functions that have been made discrete.

Figures 3 (a) and (b) show the time-course of activation for a leaky integrator unit with different values of external input $I$ and leakage parameters $\alpha$ with $I \neq 0$ for $t \in[0,20]$. In order to train a network, one needs to define an error function

$$
E=\int_{t_{0}}^{t_{1}} f_{\text {err }}[\boldsymbol{x}(t), t] \mathrm{d} t .
$$

Here we choose the least mean square function

$$
E=\frac{1}{2} \sum_{i} \int_{t_{0}}^{t_{1}} s_{i}\left[x_{i}(t)-d_{i}(t)\right]^{2} \mathrm{~d} t
$$

$d_{i}(t)$ is the desired activation of unit $i$ at time $t$ and $s_{i}$ is the relative importance of this activation where $s=0$ means unimportant and $s=1$ most important.

If one changes the activation of unit $i$ at time $t$ for a small amount, one gets a measure how much this change influences the error function:

$$
e_{i}(t)=\frac{\partial f_{e r r}[\boldsymbol{x}(t), t]}{\partial x_{i}(t)}
$$

with

$$
f_{\text {err }}=\frac{1}{2} \sum_{i} s_{i}\left[x_{i}(t)-d_{i}(t)\right]^{2} .
$$

With Eq. (9) as error function we get

$$
e_{i}(t)=s_{i}\left[x_{i}(t)-d_{i}(t)\right] .
$$

Equations (10) and (11) describe the influence of a change of activation only for $t$. In a neural net that is described by Eqs. (1) and (3) each change of activation at $t$ also influences the activation at later times $t^{\prime}\left(t<t^{\prime}\right)$. The 
amount of this influence can be described by using time-ordered derivations $[63,62]:$

$$
\begin{aligned}
\tilde{z}_{i}(t) & =\frac{\partial^{+} E}{\partial \widetilde{x_{i}}(t)} \\
& :=\frac{\partial E}{\partial \widetilde{x_{i}}(t)}+\sum_{t^{\prime}>t} \sum_{j} \frac{\partial^{+} E}{\partial \widetilde{x_{j}}\left(t^{\prime}\right)} \frac{\partial \widetilde{x_{j}}\left(t^{\prime}\right)}{\partial \widetilde{x_{i}}(t)}
\end{aligned}
$$

with

$$
\begin{array}{ll}
j=1,2, \ldots, n & n \text { number of units } \\
t^{\prime}=t+\Delta t, t+2 \Delta t, \ldots, t_{1} & t_{1} \text { last defined time }
\end{array}
$$

$\tilde{z}_{i}(t)$ measures how strong a change of activation of unit $i$ at time $t$ influences the error function for all times.

Performing the derivations in Eq. (12) with Eqs. (9), (11), (7) and (3) and setting $t^{\prime}=t+\Delta t$ gives:

$$
\begin{aligned}
\frac{\partial E}{\partial \widetilde{x}_{j}(t)} & =\Delta t e_{i} \\
\frac{\partial \widetilde{x}_{i}(t+\Delta t)}{\partial \widetilde{x_{i}}(t)} & =\left(1-\Delta t \alpha_{i}\right)+\Delta t \alpha_{i} w_{i i} f^{\prime}\left(\widetilde{y}_{i}(t)\right) \\
\frac{\partial \widetilde{x}_{j}(t+\Delta t)}{\partial \widetilde{x}_{i}(t)} & =\Delta t \alpha_{j} w_{j i} f^{\prime}\left(\widetilde{y}_{j}(t)\right)
\end{aligned}
$$

for all units $j$ that are connected with unit $i$.

All other derivations are zero. With this one gets

$$
\begin{aligned}
\widetilde{z_{i}}(t)=\Delta t e_{i} & +\left(1-\Delta t \alpha_{i}\right) \widetilde{z_{i}}(t+\Delta t) \\
& +\sum_{j} \Delta t \alpha_{j} w_{j i} f^{\prime}\left(\widetilde{y}_{j}(t)\right) \widetilde{z_{j}}(t+\Delta t) .
\end{aligned}
$$

The back-propagated error signal $\mathbf{z}(t)$ is equivalent to the $\delta$ in standard backpropagation. After the last defined activation $d_{i}\left(t_{1}\right)$ there is no further change of $E$, so $z_{i}\left(t_{1}+\Delta t\right)=0$.

Making use of Euler's method in the opposite direction one finds that the back-propagated error signal can be described by the following differential equation:

$$
\frac{\mathrm{d} z_{i}(t)}{\mathrm{d} t}=\alpha_{i} z_{i}(t)-e_{i}-\sum_{j} \alpha_{j} w_{j i} f^{\prime}\left(y_{j}(t)\right) z_{j}(t) .
$$

With Eq. (16) it is possible to calculate how the error function changes if one changes the parameters $\alpha_{i}$ and $w_{i j}$. Each variation also changes the activation $x_{i}$. The influence of this activation on $E$ can be calculated using the chain rule of derivations.

If $w_{i j}$ changes for $\Delta t$ for $\partial w_{i j}$ then the influence of this change on the error function can be described by 


$$
\begin{aligned}
\left.\frac{\partial E}{\partial w_{i j}}\right|_{t} ^{t+\Delta t} & :=\frac{\partial^{+} E}{\partial x_{i}(t+\Delta t)} \frac{\partial x_{i}(t+\Delta t)}{\partial w_{i j}} \\
& =z_{i}(t+\Delta t) \alpha_{i} x_{j}(t) f^{\prime}\left(y_{i}(t)\right) \Delta t .
\end{aligned}
$$

A change of $\partial w_{i j}$ during the whole time $t_{0} \leq t \leq t_{1}$ produces:

$$
\frac{\partial E}{\partial w_{i j}}=\alpha_{i} \int_{t_{0}}^{t_{1}} z_{i}(t) x_{j}(t) f^{\prime}\left(y_{i}(t)\right) \mathrm{d} t .
$$

For the influence of a change in $\alpha_{i}$ on $E$ one finds

$$
\begin{aligned}
\left.\frac{\partial^{+} E}{\partial \alpha_{i}}\right|_{t} ^{t+\Delta t} & =\frac{\partial E}{\partial x_{i}(t+\Delta t)} \frac{\partial x_{i}(t+\Delta t)}{\partial \alpha_{i}} \\
& \left.=z_{i}(t+\Delta t)\left\{f\left(y_{i}(t)\right)-x_{i}(t)\right)\right\} \Delta t .
\end{aligned}
$$

For the whole time:

$$
\left.\frac{\partial E}{\partial \alpha_{i}}=\int_{t_{0}}^{t_{1}} z_{i}(t)\left\{f\left(y_{i}(t)\right)-x_{i}(t)\right)\right\} \mathrm{d} t .
$$

Now we have nearly all equations that are needed for training a neural net of leaky integrator units. Finally we must keep in mind the fact that the leakage term $\alpha$ must be between 0 and 1 . This can be done by using

$$
\alpha=\frac{1}{1+e^{-\bar{\alpha}}}
$$

and learning $\bar{\alpha}$ instead of $\alpha$. With this replacement one finds

$$
\begin{aligned}
& \frac{\partial E}{\partial \overline{\alpha_{i}}}=\frac{1}{1+e^{-\overline{\alpha_{i}}}}\left(1-\frac{1}{1+e^{-\overline{\alpha_{i}}}}\right) \\
& \int_{t_{0}}^{t_{1}} z_{i}(t)\left\{f\left(y_{i}(t)\right)-x_{i}(t)\right\} \mathrm{d} t .
\end{aligned}
$$

\subsection{Overview of the learning procedure}

To start the training, one needs to have the following information:

1. topology of the net with number of units $(n)$ and connections

2. values of the parameters $\boldsymbol{w}_{\mathbf{0}}$ and $\overline{\boldsymbol{\alpha}}_{\mathbf{0}}$ at $t=0$

3. activations $\boldsymbol{x}\left(t_{0}\right)$ at $t=0$

4. time-course of the input $\left(\boldsymbol{I}^{e x t}(t), t_{0} \leq t \leq t_{1}\right)$

5. time-course of the desired output $(\boldsymbol{d}(\mathrm{t}))$

6. activation function $f$ for each unit

7. error function $E$

8. time-step size $\Delta t$ that resembles the required resolution of the time-course ( $\Delta t=0.1$ turned out to be a good default value). 
E.

The goal is to find a combination of $\mathbf{w}$ and $\overline{\boldsymbol{\alpha}}$ that gives a minimum for

1. At first one has to calculate the netinput Eq. (3) for each unit successively and for each time-step forward in time. Simultaneously, the activations are calculated with Eq. (7).

2. With Eq. (9) one calculates the main error $E$ and the error vector $\mathbf{e}(t)$ using Eq. (11).

3. Then the error signals are propagated backwards through time with Eq. (16), making use of the condition $\tilde{z}_{i}\left(t_{1}+\Delta t\right)=0$.

4. Now one calculates the gradient of each free parameter with respect to the error function $E$ with the discrete versions of Eqs. (19) and (23):

$$
\begin{aligned}
& \frac{\partial E}{\partial w_{i j}}=\frac{1}{1+e^{-\overline{\alpha_{i}}}} \sum_{t=t_{0}}^{t_{1}} \widetilde{z_{i}}(t+\Delta t) \widetilde{x_{j}}(t) f^{\prime}\left(\widetilde{y}_{i}(t)\right) \Delta t \\
& \frac{\partial E}{\partial \overline{\alpha_{i}}}= \frac{1}{1+e^{-\overline{\alpha_{i}}}}\left(1-\frac{1}{1+e^{-\overline{\alpha_{i}}}}\right) \\
& \sum_{t=t_{0}}^{t_{1}} \widetilde{z_{i}}(t)\left\{f\left(\widetilde{y_{i}}(t)\right)-\widetilde{x_{i}}(t)\right\} \Delta t .
\end{aligned}
$$

5. After this, the parameters are changed along the negative gradient (gradient descent):

$$
\begin{aligned}
w_{i j} & =w_{i j}-\eta_{w} \frac{\partial E}{\partial w_{i j}} \\
\bar{\alpha}_{i} & =\bar{\alpha}_{i}-\eta_{\bar{\alpha}} \frac{\partial E}{\partial \bar{\alpha}_{i}} .
\end{aligned}
$$

With $\eta_{w}$ and $\eta_{\bar{\alpha}}$ as learning rates. ( $\eta=0.1$ is commonly a suitable starting value.) The gradient can be used for steepest descent, conjugate gradient or other numeric approximations (see e. g. [49]).

6. Having obtained the new values $\mathbf{w}$ and $\bar{\alpha}$, the procedure goes back to step 1 and is continuously followed until the main error falls below a certain value in step 2 or this criterium is not reached after a maximal number of epochs.

(For a model that uses this type of learning algorithm for leaky integrator units see [42]). In the context of modeling oscillating brain activity, recurrent networks of leaky integrator units become interesting. Section 4 will describe a simple example. 


\section{From Physiology to Leaky Integrator Models}

\subsection{Leaky integrator model of single neurons}

Let us consider the somatic membrane of a neuron $i$ in the vicinity of the trigger zone. For the sake of simplicity we shall assume that the membrane behaves only passively at this site. For further simplification we do not describe the trigger zone by the complete Hodgkin-Huxley equations [31] but instead as a McCulloch-Pitts neuron [45], i.e. as a threshold device: the neuron fires if its membrane potential $V_{i}(t)$ exceeds a threshold $\theta$ from below due to the law of "all or nothing" [36]. Because of that the membrane potential $V_{i}(t)$ becomes translated into a spike train which can be modeled by a sum of delta functions

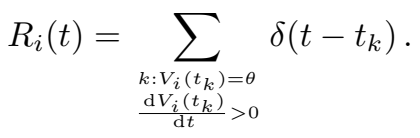

Now, we can determine the number of spikes in a time interval $[0, t][40]$ which is given by

$$
N_{i}(t)=\int_{0}^{t} R_{i}\left(t^{\prime}\right) \mathrm{d} t^{\prime},
$$

Thus, from the spike rate per unit time we regain the original signal

$$
\frac{\mathrm{d}}{\mathrm{d} t} N_{i}(t)=R_{i}(t) \text {. }
$$

In the next step, we consider the membrane potential $V_{i}$ in the vicinity of the trigger zone which obeys Kirchhoff's First Law (see Fig. 4), i.e.

$$
\sum_{j} I_{i j}=\frac{V_{i}-V_{m}}{r_{m}}+c_{i} \frac{\mathrm{d} V_{i}}{\mathrm{~d} t},
$$

here, $V_{m}$ is the Nernst equilibrium potential of the leakage channels with resistance $r_{m} . c_{i}$ is the capacitance of the membrane of neuron $i$ and $I_{i j}$ is the current through the membrane at the chosen site coming from the $j$ th synapse.

These synaptic currents depend upon both, the potential difference $\tilde{V}_{i j}-V_{i}$ between the synapse $j$ and the trigger zone of $i$ and the intracellular resistance along the current's path $r_{i j}$. Therefore

$$
I_{i j}=\frac{\tilde{V}_{i j}-V_{i}}{r_{i j}}
$$

applies. Inserting Eq. (31) into Eq. (30) yields

$$
\sum_{j} \frac{\tilde{V}_{i j}-V_{i}}{r_{i j}}=\frac{V_{i}-V_{m}}{r_{m}}+c_{i} \frac{\mathrm{d} V_{i}}{\mathrm{~d} t},
$$


and after some rearrangements

$$
r_{m} c_{i} \frac{\mathrm{d} V_{i}}{\mathrm{~d} t}+V_{i}\left(1+\sum_{j} \frac{r_{m}}{r_{i j}}\right)=V_{m}+\sum_{j} \frac{r_{m}}{r_{i j}} \tilde{V}_{i j} .
$$

After letting $V_{m}=0$ without loss of generality and introducing the time constants

$$
\tau_{i}=\frac{r_{m} c_{i}}{1+\sum_{j} \frac{r_{m}}{r_{i j}}}
$$

and provisory synaptic weights

$$
\tilde{w}_{i j}=\frac{\frac{r_{m}}{r_{i j}}}{1+\sum_{j} \frac{r_{m}}{r_{i j}}},
$$

we eventually obtain

$$
\tau_{i} \frac{\mathrm{d} V_{i}}{\mathrm{~d} t}+V_{i}=\sum_{j} \tilde{w}_{i j} \tilde{V}_{i j}
$$

Next, the postsynaptic potentials $\tilde{V}_{i j}$ require our attention. We assume that an action potential arriving at the presynaptic terminal of the neuron $j$ releases on average one transmitter vesicle. ${ }^{5}$ The content of the vesicle diffuses through the synaptic cleft and reacts with receptor molecules embedded in the postsynaptic membrane. After possibly involved chemical reactions described by kinetic differential equations [40], opened ion channels give rise to a postsynaptic pulse response potential $S_{i j}(t)$. Because we characterize the dendro-somatic membranes as linear systems here, the postsynaptic potential elicited by a spike train $R_{j}(t)$ is given by the convolution

$$
\tilde{V}_{i j}(t)=S_{i j}(t) * R_{j}(t) .
$$

Let us make a rather crude approximation here by setting the postsynaptic response function proportional to a delta function:

$$
S_{i j}(t)=g_{i j} \delta(t)
$$

where $g_{i j}$ is the gain of the synapse $j \rightarrow i$. Then the postsynaptic potential is given by the product of the gain with the spike rate of the presynaptic neuron $j$ according to Eq. (29). Finally, we must take the stochasticity of the neuron into account. This is achieved by replacing the membrane potential $V_{j}$ at the trigger zone by its average obtained from the distribution function, which leads to the characteristic sigmoidal activation functions [4], e.g. the logistic function (see Eq. (5))

5 The release of transmitter is a stochastic process that can be approximately described by a Bernoulli distribution [36], and hence, due to the limit theorem of de Moivre and Laplace, being normally distributed. 


$$
R_{j}(t)=f\left(V_{j}(t)\right)=\frac{1}{1+e^{-\beta\left[V_{j}(t)-\theta\right]}} .
$$

Collecting Eqs. (35, 36) and (38) together and introducing the proper synaptic weights

$$
w_{i j}=g_{i j} \tilde{w}_{i j},
$$

yields the leaky integrator model of a network of distributed single neurons

$$
\tau_{i} \frac{\mathrm{d} V_{i}}{\mathrm{~d} t}+V_{i}=\sum_{j} w_{i j} f\left(V_{j}(t)\right)
$$

which is analogue to Eq. (4).

\subsection{Leaky integrator model of neural populations}

According to Freeman [20] (see also [22]) a neuronal population ("KI" set) consists of many reciprocally connected neurons of one kind, either excitatory or inhibitory. Let us consider such a set of McCulloch-Pitts neurons [45] distributed over a unit volume $i$ of neural tissue. As the state variables $Q_{i}(t)$ we introduce the proportions of firing cells (either excitatory or inhibitory, in contrast to [65]) in volume $i$ at the instance of time $t[65,67]$.

A neuron belonging to volume $i$ will fire if its netinput $V_{i}$ (analogue to the membrane potential at the trigger zone, see Sec. 3.1) crosses the threshold $\theta$. But now, we have to deal with an ensemble of neurons possessing randomly distributed thresholds within the unit volume $i$. We therefore obtain an ensemble activation function [21] by integrating the corresponding probability distribution density $D(\theta)$ of thresholds [65],

$$
f\left(V_{i}\right)=\int_{0}^{V_{i}} D(\theta) \mathrm{d} \theta .
$$

Depending upon the modality of the distribution $D(\theta)$ the activation function could be a sigmoidal or even more complicated. For unimodal distributions such as Gaussian or Poissonian ones, $f\left(V_{i}\right)$ might be approximated by the logistic function Eq. (38). As for the single neuron model, the netinput is obtained by a convolution

$$
V_{i}(t)=\int_{-\infty}^{t} S\left(t-t^{\prime}\right) \sum_{j} w_{i j} Q_{j}\left(t^{\prime}\right) \mathrm{d} t^{\prime}
$$

with "synaptic weights" $w_{i j}$ characterizing the neural connectivity and whether the population is either excitatory or inhibitory.

In the following we shall simplify the model of Wilson and Cowan [65] by neglecting the refractary time. The model equations read then 


$$
Q_{i}\left(t+\tau_{i}\right)=f\left(\int_{-\infty}^{t+\tau_{i}} S\left(t-t^{\prime}\right) \sum_{j} w_{i j} Q_{j}\left(t^{\prime}\right) \mathrm{d} t^{\prime}\right)
$$

such that $Q_{i}\left(t+\tau_{i}\right)$ is the proportion of cells being above threshold in the time interval $\left[t, t+\tau_{i}\right]$. Expanding the left hand sides into a Taylor series at $t$ and assuming again that $S\left(t-t^{\prime}\right)=\delta\left(t-t^{\prime}\right)$, we obtain

$$
\tau_{i} \frac{\mathrm{d} Q_{i}(t)}{\mathrm{d} t}+Q_{i}(t)=f\left(\sum_{j} w_{i j} Q_{j}(t)\right),
$$

a leaky integrator model again, though characterized by Eq. (2).

\section{Oscillators from Leaky Integrator Units}

\subsection{Linear model}

In this section we demonstrate that a damped harmonic oscillator can be obtained from a simple model of two recurrently coupled leaky integrator units with linear activation functions. Figure 5 shows the architecture of this model.

The network of Fig. 5 is governed by the equations

$$
\begin{aligned}
& \tau_{1} \frac{\mathrm{d} x_{1}}{\mathrm{~d} t}+x_{1}=w_{11} x_{1}+w_{12} x_{2}+p \\
& \tau_{2} \frac{\mathrm{d} x_{2}}{\mathrm{~d} t}+x_{2}=w_{21} x_{1}+w_{22} x_{2}
\end{aligned}
$$

where $x_{1}$ denotes the activity of unit 1 and $x_{2}$ those of unit 2. Correspondingly, $\tau_{1}$ and $\tau_{2}$ are the time constants of the units 1 and 2 , respectively. The synaptic weights $w_{i j}$ are indicated in Fig. 5 . Note, that the weights $w_{11}$ and $w_{22}$ describe autapses [29]. The quantity $p$ refers to excitatory synaptic input that might be a periodic forcing or any other function of time.

Equations $(45,46)$ can be transformed into two second-order ordinary differential equations

$$
\begin{aligned}
& \frac{\mathrm{d}^{2} x_{1}}{\mathrm{~d} t^{2}}+\gamma \frac{\mathrm{d} x_{1}}{\mathrm{~d} t}+\omega_{0}^{2}=p_{1} \\
& \frac{\mathrm{d}^{2} x_{2}}{\mathrm{~d} t^{2}}+\gamma \frac{\mathrm{d} x_{2}}{\mathrm{~d} t}+\omega_{0}^{2}=p_{2}
\end{aligned}
$$

where we have abbreviated

$$
\gamma=\frac{\tau_{1}\left(1-w_{22}\right)+\tau_{2}\left(1-w_{11}\right)}{\tau_{1} \tau_{2}}
$$




$$
\begin{aligned}
\omega_{0}^{2} & =\frac{w_{11} w_{22}-w_{12} w_{21}-w_{11}-w_{22}+1}{\tau_{1} \tau_{2}} \\
p_{1} & =\frac{1}{\tau_{1}} \frac{\mathrm{d} p}{\mathrm{~d} t}+\frac{1-w_{22}}{\tau_{1} \tau_{2}} \\
p_{2} & =\frac{w_{21}}{\tau_{1} \tau_{2}} p
\end{aligned}
$$

Now, Eqs. $(47,48)$ describe two damped, but decoupled, harmonic oscillators with external forcing when $\gamma \geq 0$ and $\omega_{0}^{2}>0$, i.e. one unit must be excitatory and the other inhibitory.

\subsection{Simple nonlinear model}

Next, we discuss a simple nonlinear system consisting of three coupled leaky integrator units that provides a model of the thalamocortical loop. Figure 6 displays its architecture.

According to Fig. 6 the model equations read

$$
\begin{aligned}
& \tau_{1} \frac{\mathrm{d} x_{1}}{\mathrm{~d} t}+x_{1}=-\alpha f\left(x_{3}(t)\right) \\
& \tau_{2} \frac{\mathrm{d} x_{2}}{\mathrm{~d} t}+x_{2}=\beta f\left(x_{1}(t)\right) \\
& \tau_{3} \frac{\mathrm{d} x_{3}}{\mathrm{~d} t}+x_{3}=\gamma f\left(x_{2}(t)\right) .
\end{aligned}
$$

Setting all $\tau_{i}=1$ and rearrangement yields

$$
\begin{aligned}
& \frac{\mathrm{d} x_{1}}{\mathrm{~d} t}=-x_{1}-\alpha f\left(x_{3}(t)\right) \\
& \frac{\mathrm{d} x_{2}}{\mathrm{~d} t}=-x_{2}+\beta f\left(x_{1}(t)\right) \\
& \frac{\mathrm{d} x_{3}}{\mathrm{~d} t}=-x_{3}+\gamma f\left(x_{2}(t)\right) .
\end{aligned}
$$

These equations define a vector field $F$ with Jacobian matrix

$$
\mathrm{D} F=\left(\begin{array}{ccc}
-1 & 0 & -\alpha f^{\prime}\left(x_{3}(t)\right) \\
\beta f^{\prime}\left(x_{1}(t)\right) & -1 & 0 \\
0 & \gamma f^{\prime}\left(x_{2}(t)\right) & -1
\end{array}\right) .
$$

For the activation function we chose $f(x)=\tanh x$ which can be obtained by a coordinate transformation from the logistic function Eq. (38). Therefore, $F\left(x_{1}, x_{2}, x_{3}\right)=0$ and we can look whether the center manifold theorem [25] could be applied. The Jacobian at $(0,0,0)$ is

$$
\mathrm{D} F(0)=\left(\begin{array}{ccc}
-1 & 0 & -\alpha \\
\beta & -1 & 0 \\
0 & \gamma & -1
\end{array}\right)
$$


having eigenvalues

$$
\begin{aligned}
& \lambda_{1}=-1-\sqrt[3]{\alpha \beta \gamma} \\
& \lambda_{2}=-1+\frac{1}{2}(1-\mathrm{i} \sqrt{3}) \sqrt[3]{\alpha \beta \gamma} \\
& \lambda_{3}=-1+\frac{1}{2}(1+\mathrm{i} \sqrt{3}) \sqrt[3]{\alpha \beta \gamma} .
\end{aligned}
$$

Since $\lambda_{1}<0$ for $\alpha, \beta, \gamma \geq 0$, we seek for such weight parameters making $\operatorname{Re}\left(\lambda_{2 \mid 3}\right)=0$. This leads to the condition

$$
\alpha \beta \gamma=8
$$

which can be easily fulfilled, e.g. by setting

$$
\alpha=4, \quad \beta=1, \quad \gamma=2 .
$$

In this case, the center manifold theorem applies: the dynamics stabilizes along the eigenvector corresponding to $\lambda_{1}$ exhibiting a limit cycle in the center manifold spanned by the eigenvectors for $\lambda_{2}$ and $\lambda_{3}$. Figure 7 shows a numerical simulation of this oscillator.

\subsection{Random recurrent neural networks}

In the last subsection we describe a random graph carrying leaky integrator units described by Eq. (40) at its nodes. We shall see that the onset of oscillatory behavior is correlated with the emergence of super-cycles in the topology of the network provided by an evolving directed and weighted Erdős-Rényi graph of $N$ nodes where all connections between two nodes are equally likely with increasing probability $[1,12,11]$.

A directed Erdős-Rényi graph consists of a set of vertices $V$ that are randomly connected by arrows taken from an edge set $E \subset V \times V$ with equal probability $q$. The topology of the graph is completely described by its $a d$ jacency matrix $\mathbf{A}=\left(a_{i j}\right)$ where $a_{i j}=1$, if there is an arrow connecting the vertex $j$ with the vertex $i$ (i.e. $(j, i) \in E$ for $i, j \in V$ ) while $a_{i j}=0$ otherwise. A directed and weighted Erdős-Rényi graph is then described by the weight matrix $\mathbf{W}=\left(w_{i j}\right)$ which is obtained by element-wise multiplication of the adjacency matrix with constants $g_{i j}: w_{i j}=g_{i j} a_{i j}$. Biologically plausible models must satisfy Dale's law expressing that excitatory neurons have only excitatory synapses while inhibitory neurons only possess inhibitory synapses [18]. Therefore, the column vectors of the weight matrix are constrained to unique sign. We achieve this requirement by randomly choosing a proportion $p$ of the vertices to be excitatory and the remainder to be inhibitory.

In our model the weights become time-dependent due to the following evolution algorithm:

1. Initialization: $\mathbf{W}(0)=0$. 
2. At evolution time $t$, select a random pair of nodes $i, j$.

3. If they are not connected, create a synapse with weight $w_{i j}(t+1)=+\delta$ if $j$ is excitatory, and $w_{i j}(t+1)=-\delta$ if $j$ is inhibitory. If they are already connected, enhance the weight $w_{i j}(t+1)=w_{i j}(t)+\delta$ if $w_{i j}(t)>0$ and $w_{i j}(t+1)=w_{i j}(t)-\delta$ if $w_{i j}(t)<0$. All other weights remain unchanged.

4. Repeat from (ii) for a fixed number of iterations $L$.

As the "learning rate" we choose $\delta=1$ while the connectivity increases during the time $L$.

The contribution of the $i$ th neuron to the local field potential of the model is estimated by the inhomogeneity of Eq. (40),

$$
F_{i}(t)=\sum_{j} w_{i j} f\left(x_{j}\right) .
$$

Then the model EEG is given by the sum of the local field potentials of all excitatory nodes

$$
E(t)=\sum_{i^{+}} F_{i}(t)
$$

We create such random recurrent neural networks ("R2N2s") of increasing size of $N=100,200,500$, and 1000 nodes. Since about $80 \%$ of cortical neurons are excitatory pyramidal cells, $p=80 \%$ of the network's nodes are chosen to be excitatory. For each iteration of the network's evolution, the dynamics of its nodes is examined. After preparing them with normally distributed initial conditions $(\mu=0, \sigma=1)$, Eq. (40) is solved numerically for an ensemble of $K=10$ time series of length $T=100$ with a step-width of $\Delta t=0.0244$. Local field potential and EEG are computed according to Eqs. (57) and (58).

From the simulated EEGs, the power spectra are computed and averaged over all $K$ realizations of the network's dynamics. In order to monitor sudden changes in the topologies of the networks, three characteristic statistics are calculated:

(1) The mean degree (the average number of vertices attached to the nodes) $\langle k\rangle$ of the associated undirected graphs, described by the symmetrized adjacency matrix $\mathbf{A}^{s}=\left(\mathbf{A}+\mathbf{A}^{T}\right) / 2$,

(2) the total distribution

$$
d(l)=\frac{\operatorname{tr}\left(\mathbf{A}^{l}\right)}{l \mathcal{N}},
$$

of cycles of exactly length $l[1,12,11,33,9,52,56]$. In Eq. (59), $\operatorname{tr}\left(\mathbf{A}^{l}\right)$ provides the total number of (not necessarily self-avoiding) closed paths of length $l$ through the network. Since any node at such a path may serve as the starting point and there are $l$ nodes, the correct number of cycles is obtained by dividing by $l$. Finally, $\mathcal{N}=\sum_{l} \operatorname{tr}\left(\mathbf{A}^{l}\right) / l$ is a normalization constant. From the cycle distribution, we derive 
(3) an order parameter $s$ for topological transitions by the averaged slopes of the envelope of $d(l)$, where the envelopes are estimated by connecting the local maxima of $d(l)$. The above mentioned procedure is repeated for each network size $M=10$ times where we have chosen $L_{100}=150, L_{200}=400, L_{500}=$ 800 , and $L_{1000}=1700$ iterations of network evolution.

Figure 8 shows four representative networks in the critical phase characterized by the smallest positive value of the cycle order parameter $s$, averaged over the $M=10$ network simulations when sudden oscillations occur in the node's dynamics (visible in Fig. 8(a) and indicated by the peaks in the power spectra [Fig. 8(b)]). The cycle distributions $d(l)$ [Fig. 8(c)] display a transition from geometrically decaying to exponentially growing functions. As Fig. 8(b) reveals, the power spectra display a broad $1 / f$ continuum. Superimposed to this continuum are distinguished peaks that can be sloppily regarded as the "alpha waves" of the model.

According to random graph theory, Erdős-Rényi graphs exhibit a percolation transition when a giant cluster suddenly occurs for $\langle k\rangle=1[1,12,11]$. A second transition takes place for $\langle k\rangle=2$ indicating the appearance of mainly isolated cycles in the graph. Isolated cycles are characterized by a geometrically decaying envelope of the total cycle distribution. Our simulations suggest the existence of a third transition when super-cycles are composed from merging smaller ones. This is reflected by a transition of the total cycle distribution $d(l)$ from a geometrically decaying to an exponentially growing behavior due to a "combinatorial explosion" of possible self-intersecting paths through the network (super-cycles are common in regular lattices with $\langle k\rangle \geq 3$ ). We detect this transition by means of a suitably chosen order parameter $s$ derived from $d(l)$ as the averaged slope of its envelope. For decaying $d(l), s<0$ and for growing $d(l), s>0$. The appearance of super-cycles is associated with $s \approx 0$ if $d(l)$ is approximately symmetric in the range of $l$. In this case, sustained oscillations emerge in the network's dynamics due to the presence of reverberatory circles.

\section{Cognitive Modeling}

In this Chapter we have reviewed neurophysiological findings on oscillations in the electroencephalogram as well as certain approaches to model these through coupled differential equations. We have introduced into the theory of networks of leaky integrator units and presented a general learning rule to train these networks in such a way that they are able to behave in continuous time reproducing temporal patterns. This learning rule is a generalized backpropagation algorithm that has been applied for the first time to model reaction times from a psychological experiment [42]. Therefore, leaky integrator networks provide a unique and physiologically plausible paradigm for neural and cognitive modeling. 
Mathematically, leaky integrator models are described by systems of coupled ordinary differential equations which become nonlinear dynamical systems by using sigmoidal activation functions. Networks of leaky integrator units may display a realm of complex behavior: limit cycles, multistability, bifurcations and hysteresis [65]. They could therefore supply models of perceptional instability [41] or cognitive conflicts [23] as has already demonstrated by Haken [26, 27] using the so-called synergetic computers. As [27, p. 246] pointed out, the order parameter equations of synergetic computers are analogue to neural networks whose activation function is expanded into a power series. However, these computers are actually leaky integrator networks as can be seen in the following calculation.

Basically, synergetic computers are time-continuous Hopfield nets [30] governed by a differential equation

$$
\frac{\mathrm{d} \mathbf{x}}{\mathrm{d} t}-\sum_{k=1}^{K} \eta_{k} \mathbf{v}_{k}\left(\mathbf{v}_{k}^{+} \mathbf{x}\right)=-B \sum_{k^{\prime} \neq k}^{K}\left(\mathbf{v}_{k^{\prime}}^{+} \mathbf{x}\right)^{2}\left(\mathbf{v}_{k}^{+} \mathbf{x}\right) \mathbf{x}-C\left(\mathbf{x}^{+} \mathbf{x}\right) \mathbf{x}
$$

where $\mathbf{x}(t)$ denotes the activation vector of the network; the $K$ vectors $\mathbf{v}_{k}$ are training patters with adjoints $\mathbf{v}_{k}^{+}$such that orthonormality relations $\mathbf{v}_{k}^{+} \mathbf{v}_{l}=$ $\delta_{k l}$ hold. In this notation $\mathbf{x}^{+} \mathbf{y}=\sum_{i} x_{i} y_{i}$ means the inner product of the row vector $\mathbf{x}^{+}$with a column vector $\mathbf{y}$ yielding a scalar. On the other hand, the outer product $\mathbf{y} \mathbf{x}^{+}$of a column vector $\mathbf{y}$ with a row vector $\mathbf{x}^{+}$is a matrix with elements $y_{i} x_{j}$.

Therefore, the second term of the left hand side of Eq. (60) can be rewritten as

$$
\sum_{k=1}^{K} \eta_{k} \mathbf{v}_{k}\left(\mathbf{v}_{k}^{+} \mathbf{x}\right)=\sum_{k=1}^{K} \eta_{k}\left(\mathbf{v}_{k} \mathbf{v}_{k}^{+}\right) \mathbf{x}=\left(\sum_{k=1}^{K} \eta_{k} \mathbf{v}_{k} \mathbf{v}_{k}^{+}\right) \mathbf{x}=\mathbf{W} \mathbf{x}
$$

where

$$
\mathbf{W}=\sum_{k=1}^{K} \eta_{k} \mathbf{v}_{k} \mathbf{v}_{k}^{+}
$$

is the synaptic weight matrix obtained by Hebbian learning of the patterns $\mathbf{v}_{k}$ with learning rates $\eta_{k}$.

The notion "synergetic computer" refers to the possibility to describe the network (60) by the evolution of order parameters which are appropriately chosen as the "loads" of the training patters $\mathbf{v}_{k}$ in a kind of principal component analysis. We therefore separate activation space and time by the ansatz

$$
\mathbf{x}(t)=\sum_{k} \xi_{k}(t) \mathbf{v}_{k}+\mathbf{w}(t)
$$

where $\xi_{k}(t)=\mathbf{v}_{k}^{+} \mathbf{x}(t)$ and $\mathbf{w}(t)$ is a fast decaying remainder. Multiplying Eq. (60) from the left with $\mathbf{v}_{l}$ and exploiting the orthonormality relations, we eventually obtain 


$$
\frac{\mathrm{d} \xi_{l}}{\mathrm{~d} t}-\eta_{l} \xi_{l}=-B \sum_{k \neq l}^{K} \xi_{k}^{2} \xi_{l}-C\left(\sum_{k} \xi_{k}^{2}\right) \xi_{l}
$$

Dividing by $-\eta_{l}=1 / \tau_{l}$ yields then the leaky integrator equations for the order parameters with rescaled constants $B^{\prime}, C^{\prime}$ and a cubic activation function

$$
\tau_{l} \frac{\mathrm{d} \xi_{l}}{\mathrm{~d} t}+\xi_{l}=B^{\prime} \sum_{k \neq l}^{K} \xi_{k}^{2} \xi_{l}+C^{\prime}\left(\sum_{k} \xi_{k}^{2}\right) \xi_{l}
$$

The "time constants" play then the role of attention parameters describing the amount of attention devoted to a particular pattern. These parameters might also depend on time, e.g. modeling habituation.

From a formal point of view, also the attention model of Lourenço [44], the cellular neural networks of Chua [13] (see also [7, 24, 61]), the disease model of Huber et al. [32] can be regarded as leaky integrator networks.

Also higher cognitive functions such as language processing and their neural correlates such as event-related brain potentials (ERPs) [23, 8, 19] might be modeled with leaky integrator networks. Kawamoto [38] used a Hopfield net with exponentially decaying activation and habituating synaptic weights to modeling lexical ambiguity resolution. The unit's activations in his model are governed by the equations

$$
x_{i}(t+1)=f\left(\delta x_{i}(t)+\sum_{j} w_{i j} x_{j}(t)\right) .
$$

Setting $\delta=1-\alpha=1-\tau^{-1}$ and approximating $f^{\prime}(x) \approx 1$ for the typical activations, yields after a Taylor expansion

$$
f\left(\delta x_{i}(t)+\sum_{j} w_{i j} x_{j}(t)\right) \approx f\left(\sum_{j} w_{i j} x_{j}(t)\right)+f^{\prime}\left(\sum_{j} w_{i j} x_{j}(t)\right) \delta x_{i}(t)
$$

the leaky integrator equation Eq. (2).

Smolensky and Legendre [54] consider Hopfield nets of leaky integrator units that can be described by Lyapunov functions $E$. They call the function $H=-E$ the harmony of the network and argue that cognitive computations maximize this harmony function at the sub-symbolic level. Additionally, the harmony value can also be computed at the symbolic level of linguistic representations in the framework of harmonic grammars or optimality theory [50]. Regarding the harmony as an order parameter of the network would also allow to modeling neural correlates of cognitive processes, e.g., ERPs.

To conclude, leaky integrator networks provide a universal paradigm of neural and cognitive modeling with discrete units in continuous time, complementary to field theoretic approaches. 


\section{Acknowledgements}

This work has been supported by the Deutsche Forschungsgemeinschaft (research group "Conflicting Rules in Cognitive Systems"), and by the Helmholtz Institute for Supercomputational Physics at the University of Potsdam.

\section{References}

1. R. Albert and A.-L. Barabási. Statistical mechanics of complex networks. Rev. Mod. Phys., 74(1):47 - 97, 2002.

2. C. Allefeld and J. Kurths. An approach to multivariate phase synchronization analysis and its application to event-related potentials. Int. J. Bifurcation Chaos, 14(2):417 - 426, 2004.

3. C. Allefeld and J. Kurths. Testing for phase synchronization. Int. J. Bifurcation Chaos, 14(2):405 - 416, 2004.

4. D. J. Amit. Modeling Brain Function. The World of Attractor Neural Networks. Cambridge University Press, Cambridge (MA), 1989.

5. E. Başar. EEG-Brain Dynamics. Relations between EEG and Brain Evoked Potentials. Elsevier/North Holland Biomedical Press, Amsterdam, 1980.

6. E. Başar, M. Özgören, S. Karakaş, and C. Başar-Eroğlu. Super-synergy in brain oscillations and the grandmother percept is manifested by multiple oscillations. Int. J. Bifurcation Chaos, 14(2):453 - 491, 2004.

7. D. Bálya, I. Petrás, T. Roska, R. Carmona, and A. R. Vázquez. Implementing the multi-layer retinal model on the complex-cell cnn-um chip prototype. Int. J. Bifurcation Chaos, 14(2):427 - 451, 2004.

8. P. beim Graben, S. Frisch, A. Fink, D. Saddy, and J. Kurths. Topographic voltage and coherence mapping of brain potentials by means of the symbolic resonance analysis. Phys. Rev. E, 72:051916, 2005.

9. G. Bianconi and A. Capocci. Number of loops of size $h$ in growing scale-free networks. Phys. Rev. Lett., 90(7), 2003.

10. N. Birbaumer and R. F. Schmidt. Biologische Psychologie. Springer, Berlin, 3rd edition, 1996. 1st edition 1990.

11. B. Bollobás. Random Graphs, volume 73 of Cambridge Studies in Advanced Mathematics. Cambridge University Press, Cambridge (UK), 2nd edition, 2001.

12. S. Bornholdt and H. G. Schuster, editors. Handbook of Graphs and Networks. From the Genome to the Internet. Wiley-VCH, Weinheim, 2003.

13. L. O. Chua. CNN: A paradigm for complexity, volume 31 of Series on Nonlinear Science A. World Scientific, Singapore, 1998.

14. O. Creutzfeld and J. Houchin. Neuronal basis of EEG-waves. In Handbook of Electroencephalography and Clinical Neurophysiology, volume 2, Part C, pages 2C-5 - 2C-55. Elsevier, Amsterdam, 1974.

15. F. H. Lopes da Silva, A. Hoecks, H. Smits, and L. H. Zetterberg. Model of brain rhythmic activity: The alpha-rhythm of the thalamus. Kybernetik, 15:27 - 37, 1974.

16. F. H. Lopes da Silva, A. van Rotterdam, P. Bartels, E. van Heusden, and W. Burr. Models of neuronal populations: The basic mechanisms of rhythmicity. In M. A. Corner and D. F. Swaab, editors, Perspectives of Brain Research, volume 45 of Prog. Brain Res., pages 281 - 308. 1976. 
17. F. Lopes da Silva. Neural mechanisms underlying brain waves: from neural membranes to networks. Electroencephalography and Clinical Neurophysiology, 79:81 - 93, 1991.

18. P. Dayan and L. F. Abbott. Theoretical Neuroscience. Computational Neuroscience. MIT Press, Cambridge (MA), 2001.

19. H. Drenhaus, P. beim Graben, D. Saddy, and S. Frisch. Diagnosis and repair of negative polarity constructions in the light of symbolic resonance analysis. Brain and Language, 96(3):255 - 268, 2006.

20. W. J. Freeman. Mass Action in the Nervous System. Academic Press, New York (NY), 1975.

21. W. J. Freeman. Tutorial on neurobiology: From single neurons to brain chaos. Int. J. Bifurcation Chaos, 2(3):451 - 482, 1992.

22. W. J. Freeman. How and why brains create meaning from sensory information. Int. J. Bifurcation Chaos, 14(2):515 - 530, 2004.

23. S. Frisch, P. beim Graben, and M. Schlesewsky. Parallelizing grammatical functions: P600 and P345 reflect different cost of reanalysis. Int. J. Bifurcation Chaos, 14(2):531 - 549, 2004.

24. V. Gál, J. Hámori, T. Roska, D. Bálya, Zs. Borostyánköi, M. Brendel, K. Lotz, L. Négyessy, L. Orzó, I. Petrás, Cs. Rekeczky, J. Takács, P. Venetiáner, Z. Vidnyánszky, and Á Zarándy. Receptive field atlas and related cnn models. Int. J. Bifurcation Chaos, 14(2):551 - 584, 2004.

25. J. Guckenheimer and P. Holmes. Nonlinear Oscillations, Dynamical Systems, and Bifurcations of Vector Fields, volume 42 of Springer Series of Appl. Math. Sciences. Springer, New York, 1983. 5th reprint 1997.

26. H. Haken. Synergetic Computers and Cognition. A top-down Approach to Neural Nets, volume 50 of Springer Series in Synergetics. Springer, Berlin, 1991.

27. H. Haken. Principles of Brain Functioning, volume 67 of Springer Series in Synergetics. Springer, Berlin, 1996.

28. D. O. Hebb. The Organization of Behavior. Wiley, New York (NY), 1949.

29. C. S. Herrmann and A. Klaus. Autapse turns neuron into oscillator. Int. J. Bifurcation Chaos, 14(2):623 - 633, 2004.

30. J. Hertz, A. Krogh, and R. G. Palmer. Introduction to the Theory of Neural Computation, volume I of Lecture Notes of the Santa Fe Institute Studies in the Science of Complxity. Perseus Books, Cambridge (MA), 1991.

31. A. L. Hodgkin and A. F. Huxley. A quantitative description of membrane current and its application to conduction and excitation in nerve. J. Physiol., 117:500 $-544,1952$.

32. M. T. Huber, H. A. Braun, and J.-C. Krieg. Recurrent affective disorders: nonlinear and stochastic models of disease dynamics. Int. J. Bifurcation Chaos, 14(2):635 - 652, 2004.

33. S. Itzkovitz, R. Milo, N. Kashtan, G. Ziv, and U. Alon. Subgraphs in random networks. Phys. Rev. E, 68, 2003.

34. V. K. Jirsa. Information processing in brain and behavior displayed in largescale scalp topographies such as EEG and MEG. Int. J. Bifurcation Chaos, 14(2):679 - 692, 2004.

35. V. K. Jirsa and H. Haken. Field theory of electromagnetic brain activity. Phys. Rev. Lett., 77(5):960 - 963, 1996.

36. E. R. Kandel, J. H. Schwartz, and T. M. Jessel, editors. Principles of Neural Science. Appleton \& Lange, East Norwalk, Connecticut, 3rd edition, 1991. 
37. S. Kaplan, M. Sonntag, and E. Chown. Tracing recurrent activity in cognitive elements (TRACE): A model of temporal dynamics in a cell assembly. Connection Science, 3:179 - 206, 1991.

38. A. H. Kawamoto. Nonlinear dynamics in the resolution of lexical ambiguity: A parallel distributed processing account. Journal of Memory and Language, 32:474 - 516, 1993.

39. W. Klimesch, M. Schabus, M. Doppelmayr, W. Gruber, and P. Sauseng. Evoked oscillations and early components of event-related potentials: An analysis. Int. J. Bifurcation Chaos, 14(2):705 - 718, 2004.

40. C. Koch and I. Segev, editors. Methods in Neuronal Modelling. From Ions to Networks. Computational Neuroscience. MIT Press, Cambridge (MA), 2nd edition, 1998.

41. J. Kornmeier, M. Bach, and H. Atmanspacher. Correlates of perceptive instabilities in visually evoked potentials. Int. J. Bifurcation Chaos, 14(2):727-736, 2004.

42. T. Liebscher. Modeling reaction times with neural networks using leaky integrator units. In K. Jokinen, D. Heylen, and A. Nijholt, editors, Proc. 18th Twente Workshop on Language Technology, volume 18 of TWLT, pages 81 - 94, Twente (NL), 2000. Univ. Twente.

43. D. T. J. Liley, D. M. Alexander, J. J. Wright, and M. D. Aldous. Alpha rhythm emerges from large-scale networks of realistically coupled multicompartmental model cortical neurons. Network: Comput. Neural Syst., 10:79 - 92, 1999.

44. C. Loureno. Attention-locked computation with chaotic neural nets. Int. J. Bifurcation Chaos, 14(2):737 - 760, 2004.

45. W. S. McCulloch and W. Pitts. A logical calculus of ideas immanent in nervous activity. Bull. Math. Biophys., 5:115 - 133, 1943.

46. P. L. Nunez. Electric Fields of the Brain: The Neurophysics of EEG. Oxford University Press, New York, 1981.

47. B. A. Pearlmutter. Gradient calculations for dynamic recurrant neural networks: A survey. IEEE Trans. Neural Networks, 6(5):1212 - 1228, 1995.

48. G. Pfurtscheller. EEG rhythms - event related desynchronization and synchronization. In H. Haken and H. P. Koepchen, editors, Rhythms in Physiological Systems, volume 55 of Springer Series in Synergetics, pages 289 - 296, Berlin, 1991. Springer.

49. W. H. Press, S. A. Teukolsky, W. T. Vetterling, and B. P. Flannery. Numerical Recipies in C. Cambridge University Press, New York, 2nd 1992 edition, 1996. reprinted 1996.

50. A. Prince and P. Smolensky. Optimality: From neural networks to universal grammar. Science, 275:1604-1610, 1997.

51. P. A. Robinson, C. J. Rennie, J. J. Wright, H. Bahramali, E. Gordon, and D. L. Rowe. Prediction of electroencephalic spectra from neurophysiology. Phys. Rev. E, 63, 2001. 021903.

52. H. D. Rozenfeld, J. E. Kirk, E. M. Bollt, and D. ben Avraham. Statistics of cycles: How loopy is your network? J. Phys. A: Math. Gen., 38:4589-4595, 2005.

53. D. E. Rumelhart, J. L. McClelland, and the PDP Research Group, editors. Parallel Distributed Processing: Explorations in the Microstructure of Cognition, volume I. MIT Press, Cambridge (MA), 1986. 
54. P. Smolensky and G. Legendre. The Harmonic Mind. From Neural Computation to Optimality-Theoretic Grammar, volume 1. Cognitive Architecture. MIT Press, Cambridge (MA), 2006.

55. E.-J. Speckmann and C. E. Elger. Introduction to the neurophysiological basis of the EEG and DC potentials. In E. Niedermeyer and F. Lopez da Silva, editors, Electroencephalography. Basic Principles, Clinical Applications, and Related Fields, chapter 2, pages 15 - 27. Lippincott Williams and Wilkins, Baltimore, 4th edition, 1999.

56. O. Sporns, G. Tononi, and G. M. Edelman. Theoretical neuroanatomy: Relating anatomical and functional connectivity in graphs and cortical connection matrices. Cerebral Cortex, 10(2):127 - 141, 2000.

57. R. Srinivasan. Internal and external neural synchronization during conscious perception. Int. J. Bifurcation Chaos, 14(2):825 - 842, 2004.

58. M. Steriade. Cellular substrates of brain rhythms. In E. Niedermeyer and F. Lopez da Silva, editors, Electroencephalography. Basic Principles, Clinical Applications, and Related Fields, chapter 3, pages 28 - 75. Lippincott Williams and Wilkins, Baltimore, 4th edition, 1999.

59. M. Steriade, P. Gloor, R. R. Llinás, F. H. Lopes da Silva, and M.-M. Mesulam. Basic mechanisms of cerebral rhythmic activities. Electroencephalography and Clinical Neurophysiology, 76:481 - 508, 1990.

60. A. van Rotterdam, F. H. Lopes da Silva, J. van den Ende, M. A. Viergever, and A. J. Hermans. A model of the spatial-temporal characteristics of the alpha rhythm. Bull. Math. Biol., 44(2):283 - 305, 1982.

61. F. S. Werblin and B. M. Roska. Parallel visual processing: A tutorial of retinal function. Int. J. Bifurcation Chaos, 14(2):843 - 852, 2004.

62. P. Werbos. Maximizing long-term gas industry profits in two minutes in Lotus using neural network models. IEEE Trans. on Systems, Man, and Cybernetics, 19:315 - 333, 1989.

63. P. Werbos. Back-propagation through time: What it does and how to do it. volume 78 of Proc. IEEE, pages 1550 - 1560, ?, 1990.

64. H. R. Wilson. Spikes, Decisions and Actions. Dynamical Foundations of Neuroscience. Oxford University Press, New York (NY), 1999.

65. H. R. Wilson and J. D. Cowan. Excitatory and inhibitory interactions in localized populations of model neurons. Biophys. J., 12:1-24, 1972.

66. J. J. Wright and D. T. J. Liley. Dynamics of the brain at global and microscopic scales: Neural networks and the EEG. Behavioral and Brain Sciences, 19:285 320, 1996.

67. J. J. Wright and D. T. L. Liley. Simulation of electrocortical waves. Biological Cybernetics, 72:347 - 356, 1995.

68. J. J. Wright, C. J. Rennie, G. J. Lees, P. A. Robinson, P. D. Bourke, C. L. Chapman, E. Gordon, and D. L. Rowe. Simulated electrocortical activity at microscopic, mesoscopic, and global scales. Neuropsychopharmacology, 28:S80 S93, 2003.

69. J.J. Wright, C.J. Rennie, G.J. Lees, P.A. Robinson, P.D. Bourke, C.L. Chapman, E. Gordon, and D.L. Rowe. Simulated electrocortical activity at microscopic, mesoscopic and global scales. Int. J. Bifurcation Chaos, 14(2):853 - 872, 2004.

70. S. Zschocke. Klinische Elektroenzephalographie. Springer, Berlin, 1995. 
beim Graben et al.

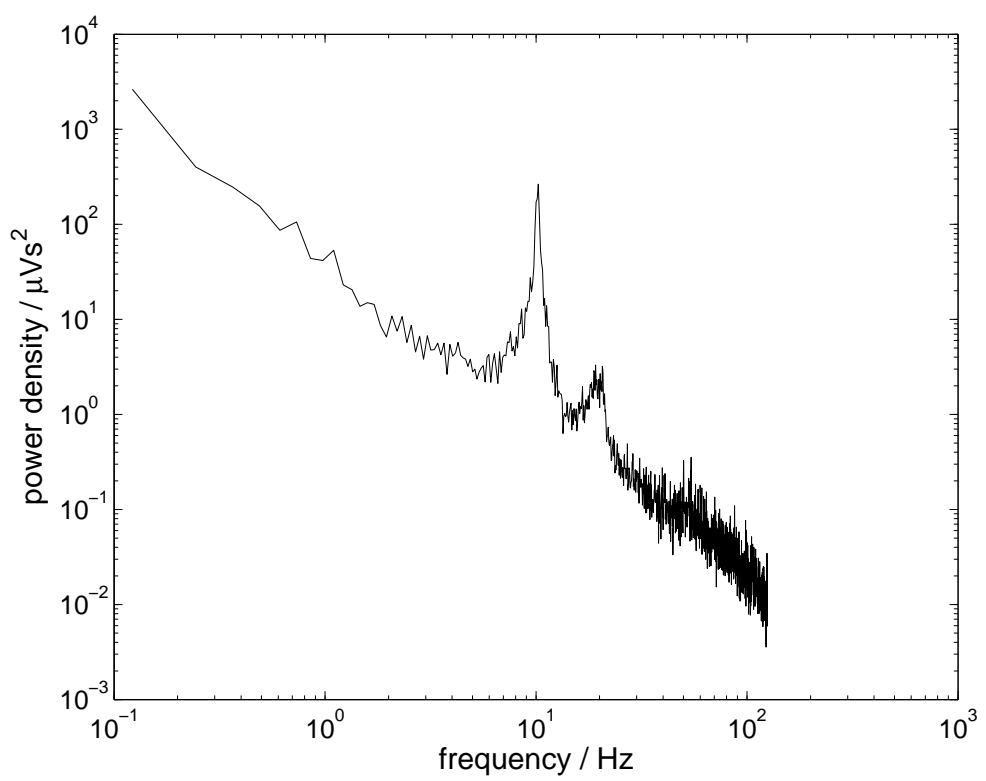

Fig. 1. Power spectrum of the alpha EEG at one parietal electrode site (PZ). 
(a)

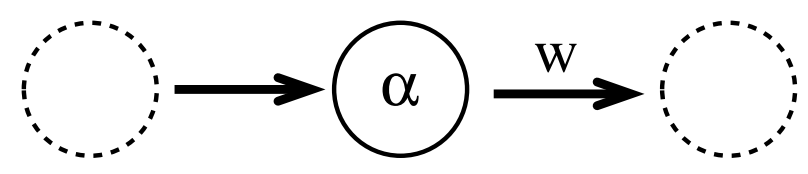

(b)

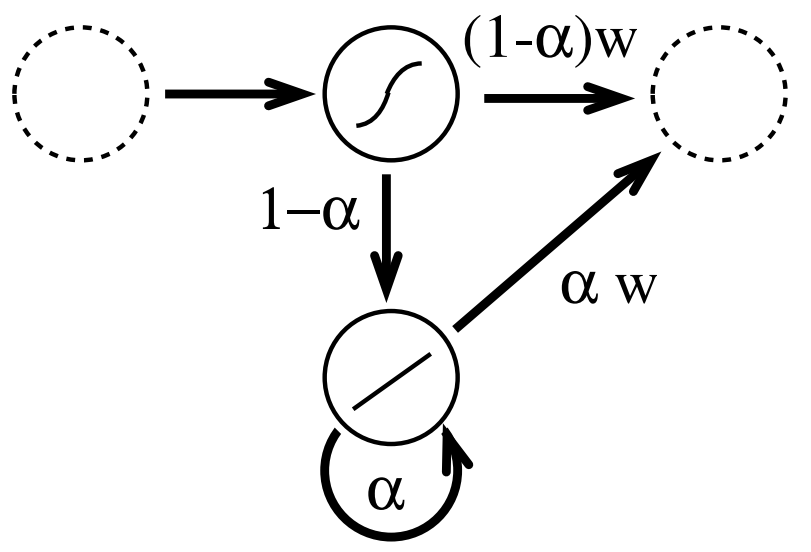

Fig. 2. Simulation of a leaky integrator unit (a) and a recurrent combination of two standard units (b). The function of the leakage term $\alpha$ is mimicked by two parallel standard units with a logistic and a linear activation function, respectively. 


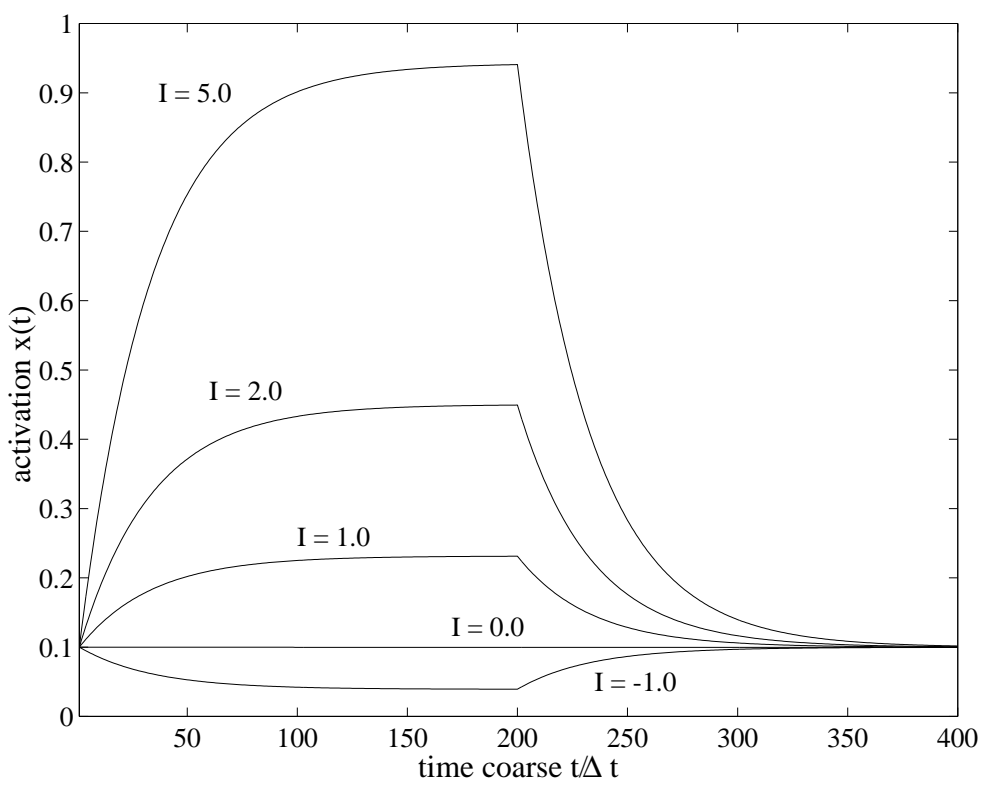

(a)

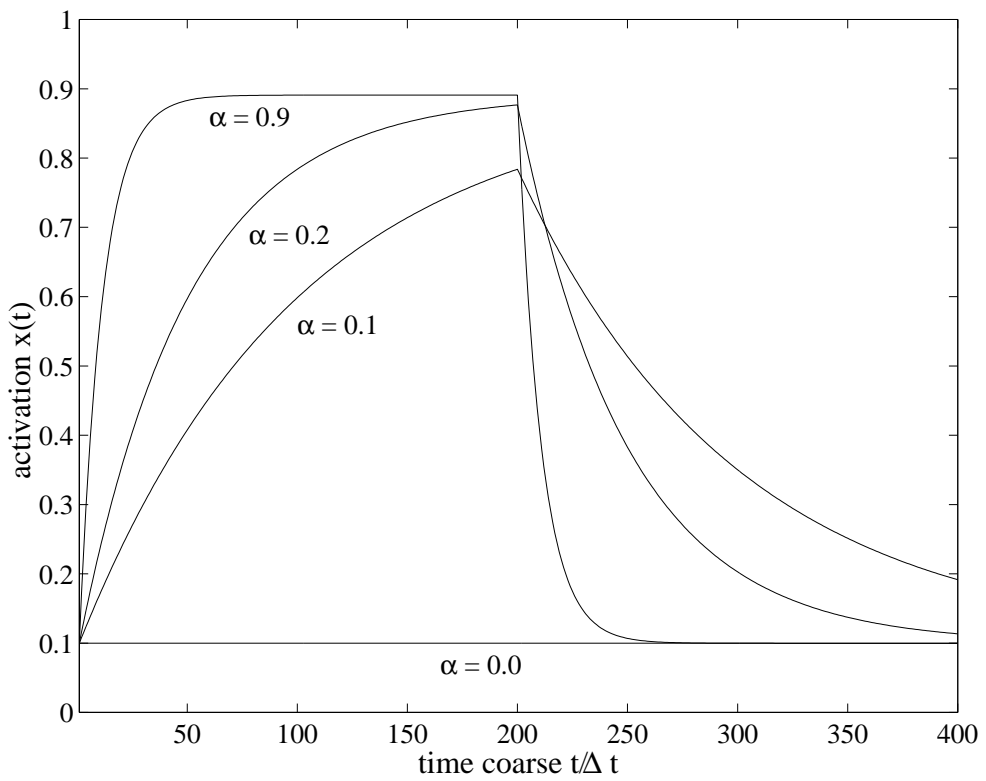

(b)

Fig. 3. Time-course of activation (a)for different input with $\Delta t=0.1, \alpha=0.3$ and $b=-2.2$; (b) Time-course of activation for different leakage rates with $\Delta t=0.1$, $I^{e x t}=4.3$ und $b \approx-2.2$. 


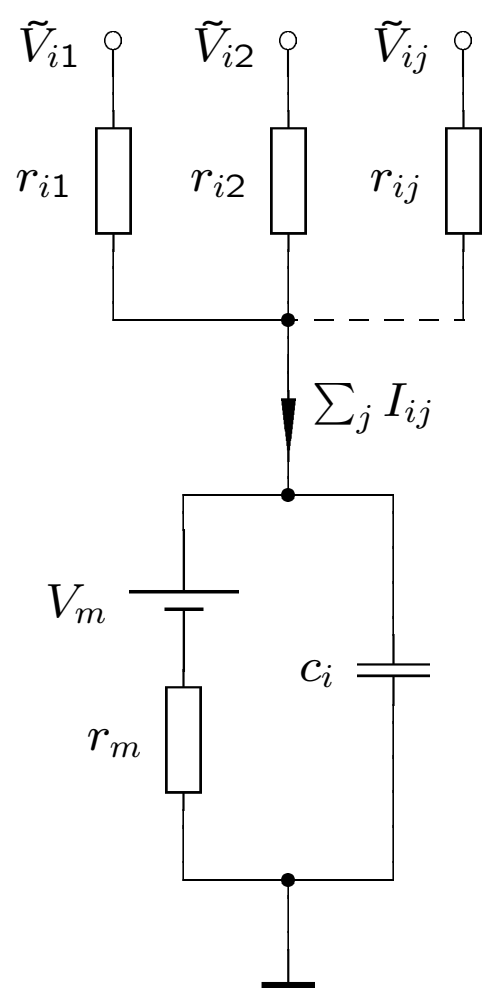

Fig. 4. Equivalent circuit for the leaky integrator neuron.

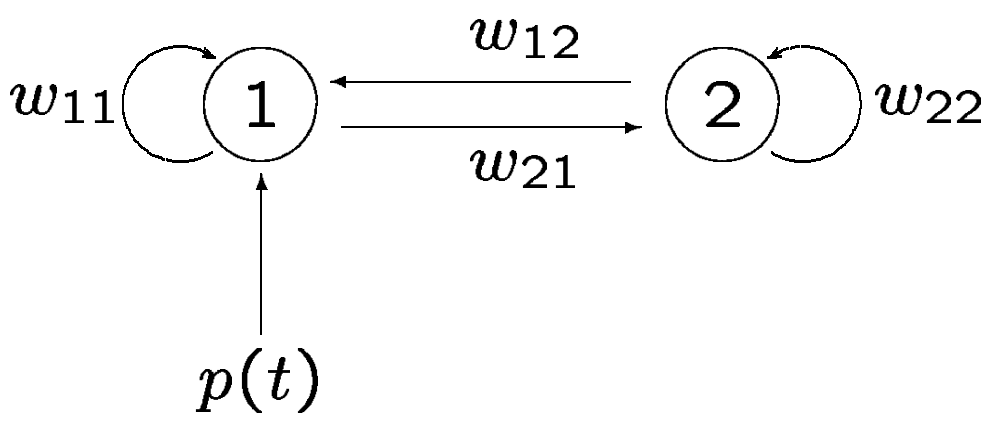

Fig. 5. Architecture of an oscillator formed by leaky integrator units. 


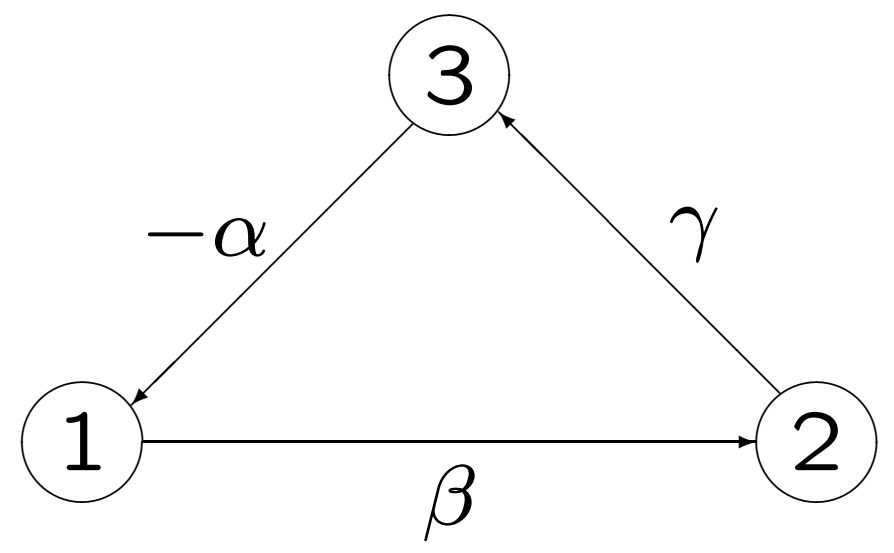

Fig. 6. Thalamocortical oscillator of three leaky integrator units: 1 pyramidal cell; 2 thalamus cell, 3 cortical star cell.

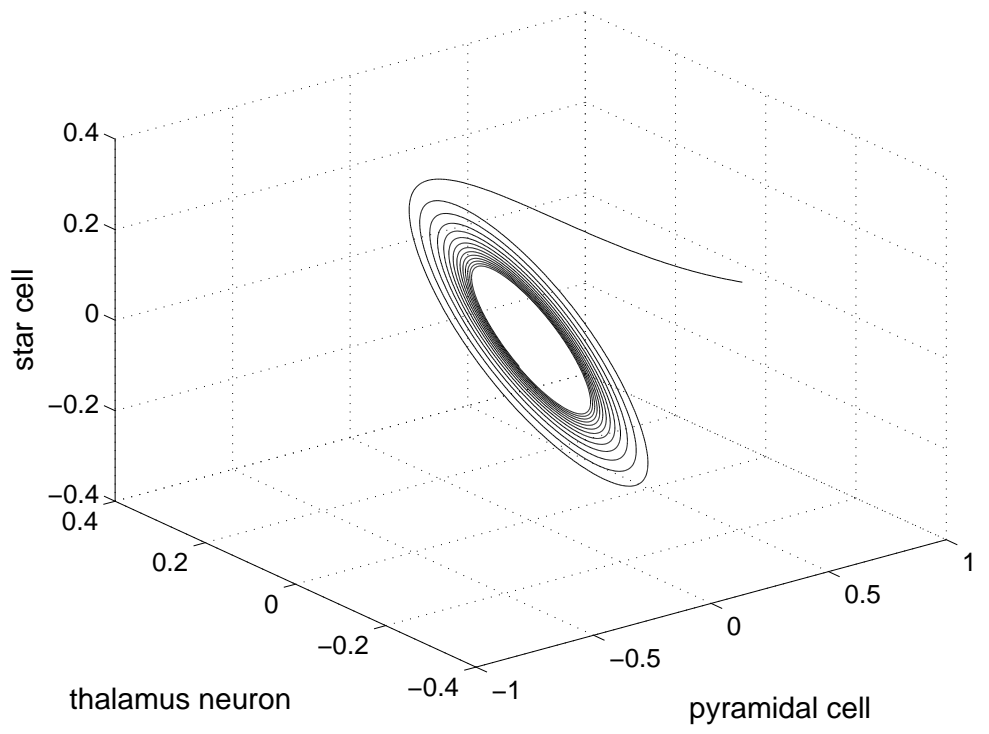

Fig. 7. Limit cycle of the thalamocortical oscillator in its center manifold plane. 


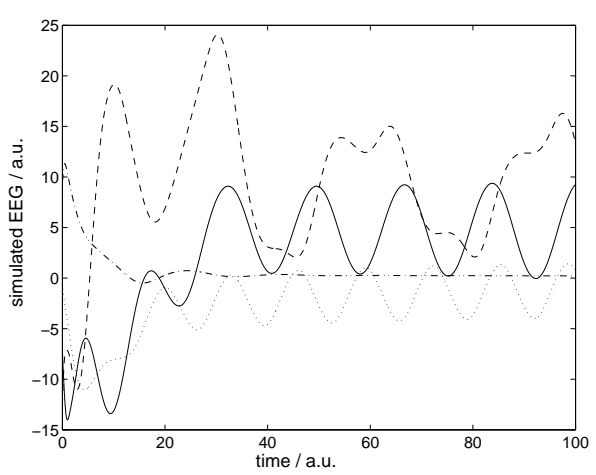

(a)

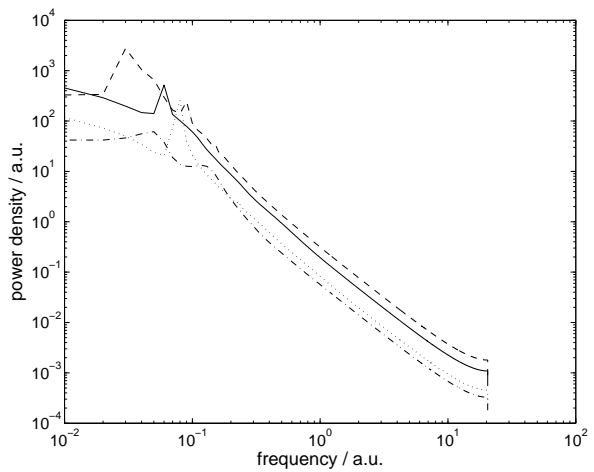

(b)

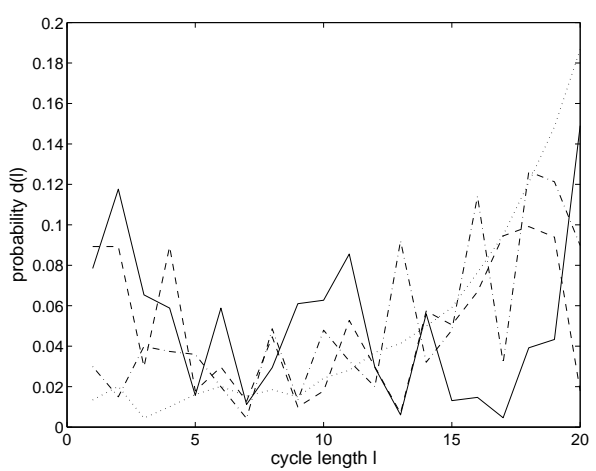

(c)

Fig. 8. (a) Representative simulated time series during the oscillatory transition (critical phase) for four different network sizes: $N=100$ (dotted), $N=200$ (dasheddotted), $N=500$ (solid), and $N=1000$ (dashed). (b) Power spectra of simulated time series. (c) Total distributions of cycles [Eq. (59)] for the same networks. 



\section{Index}

$1 / f, 2,17$

activation, 4

activation function, 2, 4, 8, 11, 13, 19

activation vector, 18

adjacency matrix, 15

alpha blocking, 2, 3

alpha waves, 2, 3, 17

ambiguity resolution, 19

attention parameter, 19

attractor, 5

autapse, 13

backpropagation, 5, 7, 17

beta waves, 2

bifurcations, 18

cell assembly, 3, 4

cellular neural networks, 19

center manifold, 15

center manifold theorem, 14, 15

cognitive conflicts, 18

cognitive processes, 2,3

convolution, 11

cycle distribution, 16, 17

Dale's law, 15

depolarization, 1

desynchronization, 2

difference equation, 6

differential equation, 5-7, 13, 17, 18

EEG, 1, 16

eigenvector, 15 electroencephalogram, 1, 17

Erdős-Rényi graph, 15, 17

ERP, 19

error function, 6-8

Euler's method, 6, 7

event-related brain potentials, 19

excitatory synapse, 1, 15

gradient descent, 9

habituation, 19

harmonic grammar, 19

harmonic oscillator, 13, 14

harmony function, 19

Hebbian learning, 18

higher cognitive functions, 19

Hodgkin-Huxley equations, 3, 10

Hopfield network, 5, 18, 19

hyperpolarization, 1

hysteresis, 18

inhibitory synapse, 1,15

input, 8

isolated cycles, 17

Jacobian matrix, 14

Kirchhoff's First Law, 10

language processing, 19

leakage, 4, 6, 8

leaky integrator networks, 4, 5, 17, 19

leaky integrator neuron, 27

leaky integrator unit, 4-6, 8, 9, 15, 17, 19 
learning rate, $9,16,18$

learning rule, 5,17

limit cycle, 2, 18

linguistic representations, 19

local field potential, 1, 16

logistic function, 5, 14

Lyapunov functions, 19

McCulloch-Pitts neuron, 2, 10, 12

mean degree, 16

membrane, 10

membrane potential, 10, 12

multistability, 18

Nernst equilibrium potential, 10 netinput, 4, 9, 12

network evolution, $3,15,17$

neural correlates, 19

neural field theories, 3

neural networks, 2, 5, 8, 18

neural oscillations, 4, 17

neuron, 10

neuronal population, 12

optimality theory, 19

order parameter, 17-19

orthonormality, 18

output, 8

perceptional instability, 18

percolation transition, 17

periodic forcing, 13

postsynaptic potential, $2,3,11$

potential difference, 10

power spectrum, 2, 16, 17

principal component analysis, 18

pyramidal cell, 1, 16, 28 random graph, 15

random graph theory, 17

random recurrent neural networks, 15, 16

reaction times, 17

recurrent networks, 5, 9

reverberatory circles, 3,17

spatio-temporal dynamics, 3

spike rate, $2,3,10$

spike train, 10, 11

star cell, 28

sub-symbolic, 3, 19

super-cycles, 15, 17

symbolic, 19

synapse, 10

synaptic weights, 11-13, 18, 19

synchronziation, 2

synergetic computer, 18

Taylor series, 13, 19

thalamocortical loop, 2, 14

thalamocortical oscillator, 28

thalamus, 28

time constant, 4, 19

time-ordered derivations, 7

topological transitions, 17

topology, 8, 15

training, 8

transmitter vesicle, 11

trigger zone, 10, 12

vector field, 14

wakefulness, 2

weight, 5

weight matrix, 15 
